\title{
Case Report - The role of somatostatin analogue therapy in nesidioblastosis following Roux-en-Y bypass surgery
}

\author{
Rajamani Manikandan, Shiva Mongolu, Azraai Nasruddin
}

Endocrinology Department, Southampton General Hospital, Southampton, UK

\begin{abstract}
Background
Postprandial hyperinsulinemic hypoglycaemia due to nesidioblastosis is a significant and debilitating complication after Roux-en-Y gastric bypass surgery (RYGB). There is growing evidence suggesting this is due to increased incretin hormones secretion. Nesidioblastosis can be difficult to manage with variable response to different pharmacological therapies and some requiring partial/total pancreatectomy or revision of bypass surgery pouch. We present a patient who has had partial response to octreotide.
\end{abstract}

\section{Case report}

A 45-year-old lady had RYGB 18months ago for significant reflux symptoms. She also lost $35 \mathrm{~kg}$ but developed severe postprandial neuroglycopenic symptoms that occurred 90 to 120 minutes after meals. She gained $10 \mathrm{~kg}$ in weight.

During oral glucose tolerance test (OGTT) her blood glucose dropped to $1 \mathrm{mmol} / \mathrm{l}$ at 90 minutes with Insulin $11.1 \mathrm{mu} / \mathrm{L}$ and C-peptide $2595 \mathrm{pmol} / \mathrm{I}$. She did not have any benefit from dietetic advice, low Gl diet, acarbose and Guar gum. Pancreatic CT scans and calcium stimulation test did not localize a cause for her hyperinsulinemia. Her symptoms improved partially with diazoxide but it was withdrawn due to side effects. Repeating OGTT on diazoxide, her glucose dropped to $1.4 \mathrm{mmol} / \mathrm{L}$ in 90 minutes. Her symptoms improved with octreotide 50micrograms TDS. OGTT done after 6 months on octreotide therapy showed peak glucose of $14.9 \mathrm{mmol} / \mathrm{L}$ at 90 minutes with blunted insulin response with peak levels of $33.5 \mathrm{mu} / \mathrm{L}$. However, she continued to report intermittent postprandial hypoglycaemia. We switched her to octreotide LAR but hypoglycaemia got worse. Her symptoms are currently well controlled on Pasereotide $200 \mathrm{mcg} /$ day.

\section{OGTT Results}
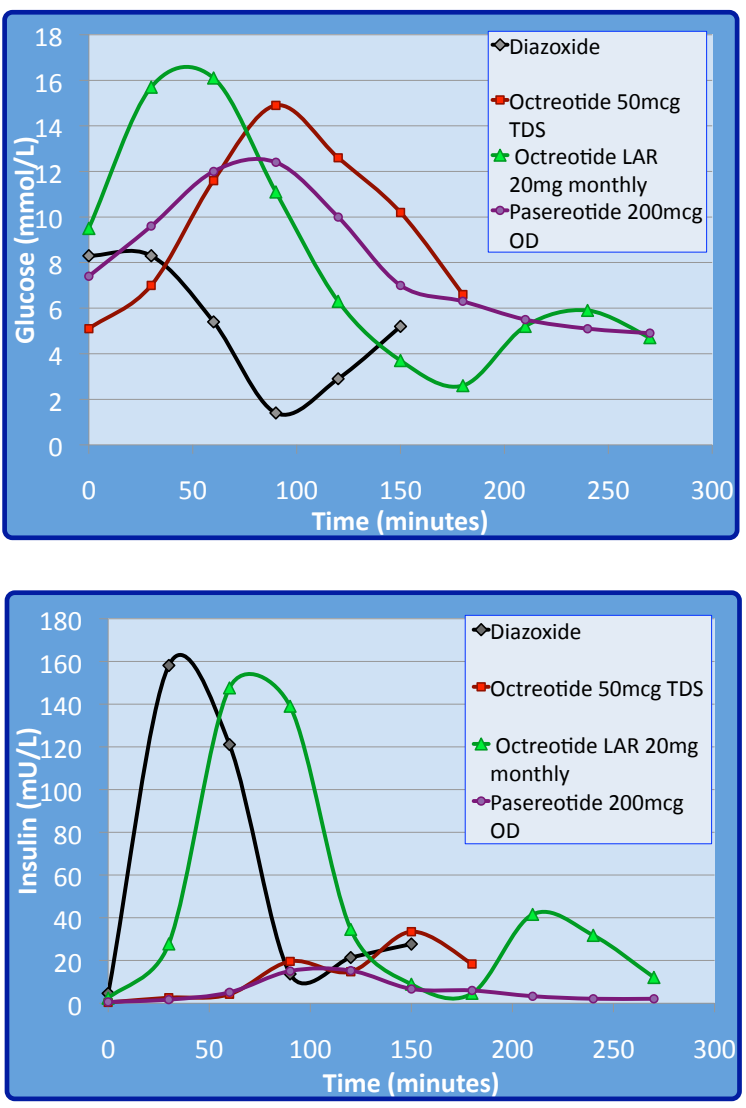

\section{Discussion}

Our case highlights the difficulties in managing the symptoms of nesidioblastosis. Subcutaneous octreotide worked well initially but the effects became attenuated. Her symptoms could be episodes of rebound hypoglycaemia when octreotide effect wore off. It is unclear why octreotide LAR did not work. Pasireotide with its potent suppression of incretin release is keeping her symptoms under control. We have obtained funding for a trial of pasireotide therapy. 